\title{
In vivo multiphoton imaging reveals gradual growth of newborn amyloid plaques over weeks
}

\author{
Steffen Burgold • Tobias Bittner • Mario M. Dorostkar • Daniel Kieser • \\ Martin Fuhrmann • Gerda Mitteregger • Hans Kretzschmar • \\ Boris Schmidt · Jochen Herms
}

Received: 1 October 2010/Revised: 25 November 2010/Accepted: 27 November 2010/Published online: 7 December 2010

(C) The Author(s) 2010. This article is published with open access at Springerlink.com

\begin{abstract}
The kinetics of amyloid plaque formation and growth as one of the characteristic hallmarks of Alzheimer's disease $(\mathrm{AD})$ are fundamental issues in AD research. Especially the question how fast amyloid plaques grow to their final size after they are born remains controversial. By long-term two-photon in vivo imaging we monitored individual methoxy-X04-stained amyloid plaques over 6 weeks in 12 and 18 months old Tg2576 mice. We found that in 12 months old mice, newly appearing amyloid plaques were initially small in volume and subsequently grew over time. The growth rate of plaques was inversely proportional to their volume; thus amyloid plaques that were already present at the first imaging time point grew over time but slower compared to new plaques. Additionally, we analyzed 18 months old Tg2576 mice in which we neither found newly appearing plaques nor a significant growth of pre-existing plaques over 6 weeks of imaging. In conclusion, newly appearing amyloid plaques are initially small in size but grow over time until plaque growth can not be detected anymore in aged mice. These results
\end{abstract}

Electronic supplementary material The online version of this article (doi:10.1007/s00401-010-0787-6) contains supplementary material, which is available to authorized users.

S. Burgold · T. Bittner - M. M. Dorostkar - M. Fuhrmann ·

G. Mitteregger · H. Kretzschmar · J. Herms $(\square)$

Center of Neuropathology and Prion Research,

Ludwig Maximilians Universität, Feodor-Lynen-Str. 23,

81377 Munich, Germany

e-mail: jochen.herms@med.uni-muenchen.de

D. Kieser · B. Schmidt

Clemens Schoepf Institute for Organic Chemistry

and Biochemistry, Technical University,

64287 Darmstadt, Germany suggest that drugs that target plaque formation should be most effective early in the disease, when plaques are growing.

Keywords Alzheimer's disease - Amyloid plaque . $\mathrm{A} \beta$, plaque growth $\cdot \mathrm{Tg} 2576 \cdot$ Two-photon · In vivo . Imaging

\section{Introduction}

Amyloid plaques represent one of the major hallmarks of Alzheimer's disease (AD) pathology. They are comprised of fibrillar aggregates of the amyloid- $\beta$-peptide which has been shown to have synaptotoxic effects $[10,17,18,23]$. In $\mathrm{AD}$ patients as well as in animal models synapse loss is most prominent in close proximity to amyloid plaque deposits which indicates a link between amyloid plaques and synaptotoxicity [7, 14, 15, 19-21]. Therefore, elucidating the process of amyloid plaque formation and growth may reveal important targets for therapeutic interventions to stop the progression of synapse loss and consequently cognitive decline in AD.

In vitro studies of plaque formation established a model in which a seed of fibrillar $A \beta$ is formed first and plaques grow subsequently around these seeds in a time- and concentration-dependent manner $[4,11]$. These findings were corroborated by in vivo imaging studies of $A D$ transgenic (APP/PS1) mice, which reported a change in size of a subset of plaques [2] or a gradual growth of almost all plaques, which was faster in smaller plaques and younger animals [24]. These findings, however, are contrasted by different studies, which reported that $\mathrm{A} \beta$ plaques appeared rapidly in both APP/PS1 and Tg2576 transgenic mice [16] and that these were relatively stable over time in 
Tg2576 transgenic mice [3]. The apparent discrepancies of the in vivo studies may be attributed to a different preparation of the cranial window (e.g., thinned skull vs. open skull preparations), the age of the mice, or different disease models (e.g., APP/PS1 or Tg2576). In order to reconcile these findings, we studied the appearance of $A \beta$ plaques in detail. We followed plaque formation in 12 and 18 months old Tg2576 mice by measuring the volume, as well as area, over a period of 6 weeks. While we used an open skull preparation, we started our imaging protocol 3 weeks after surgery, so that possible artifacts from inflammatory reactions [8] were minimized.

Using this approach, we found that plaques appeared and increased in volume in 12 months old, but not in 18 months old mice. Nascent plaques were initially small in volume and grew over time. Pre-existing plaques, in contrast, had a larger volume and grew slower compared to newly formed ones. These findings demonstrate that $A \beta$ plaques grow in a size-dependent manner in young (12 months) mice, while no growth was observed in older (18 months) mice.

\section{Materials and methods}

Transgenic mice

Heterozygous $\mathrm{Tg} 2576$ [9] mice (B6;SJL-Tg(APPSWE) 2576Kha from Taconic, Cologne, Germany) were crossed with heterozygous YFP-H [5] mice (B6.Cg-Tg(Thy1YFPH)2Jrs/J) from The Jackson Laboratory, Bar Harbor, USA) expressing yellow fluorescent protein (YFP) in a subset of cortical neurons. YFP-labeled neurons served as a counterstaining to find the same imaging positions over and over again in subsequent time points. Mice were of both sexes and were group housed under pathogen-free conditions until surgery, after which they were singly housed. All procedures were performed in accordance with an animal protocol approved by the University of Munich and the Government of Upper Bavaria (55.2-1.54-2531110-06).

\section{Open-skull surgery}

A cranial window over the right cortical hemisphere was surgically implanted as previously described $[1,6,8]$. The mice were anesthetized with an intraperitoneal injection of ketamine/xylazine $(0.13 / 0.01 \mathrm{mg} / \mathrm{g}$ body weight $)$. Additionally, dexamethasone $(0.02 \mathrm{ml}$ at $4 \mathrm{mg} / \mathrm{ml})$ was intraperitoneally administered immediately before surgery (Holtmaat et al. 2005). A circular piece of the skull (4 mm in diameter) over the right somatosensory cortex (centered over the parietal bone, approx. $5.5 \mathrm{~mm}$ caudal from the bregma and $5.5 \mathrm{~mm}$ lateral of the midline) was removed using a dental drill (Schick-Technikmaster C1; Pluradent; Offenbach, Germany). Immediately, a round coverslip ( $5 \mathrm{~mm}$ diameter) was glued to the skull using dental acrylic (Cyano-Veneer fast; Heinrich Schein Dental Depot, Munich, Germany) to close the craniotomy. A small metal bar, containing a hole for a screw, was glued next to the coverslip to allow repositioning of the mouse during subsequent imaging sessions. After surgery, mice received a subcutaneous analgesic dose of carprophen (Rimadyl; Pfizer, New York, USA) for 3 days $(5 \mathrm{mg} / \mathrm{kg})$. Imaging began following a 21 day rest period after surgery.

\section{Long-term two-photon in vivo imaging}

Long-term two-photon imaging was performed as previously described $[1,6]$. Less than $50 \mathrm{~mW}$ laser power was delivered to the tissue to avoid laser-induced phototoxicity. For amyloid plaque staining, methoxy-X04 [13] was intraperitoneally injected $24 \mathrm{~h}$ before every imaging session. Initially, we administered doses of $2.4 \mathrm{mg} / \mathrm{kg}$. However, in an attempt to reduce methoxy-X04 usage, we changed the dosing regimen to an initial loading dose of $2.0 \mathrm{mg} / \mathrm{kg}$ before the first imaging session and subsequent weekly maintenance doses of $0.4 \mathrm{mg} / \mathrm{kg}$, which did not affect the signal-to-noise ratio (data not shown). Furthermore, we found no significant difference in plaque growth between these two dosing regimens (Supplementary Fig. S1a), therefore we pooled the data for all further analyses. To determine whether repeated administration of methoxy-X04 increased the apparent volume of plaques by increasing the brightness, we correlated plaque volumes with the mean intensity of the fluorescence values of all voxels in a plaque (Supplementary Fig. S1b). The correlation between plaque volume and intensity was weak $\left(R^{2}=0.25\right)$ compared to data obtained by imaging fluorescent beads with varying laser intensities to simulate increasing brightness $\left(R^{2}=0.71\right.$; data not shown). Considering that no plaque growth was observed in older animals despite the same dosing scheme and that comparably little plaque growth was observed with daily administration of methoxy-X04 (Supplementary Fig. S2), changes in plaque volumes cannot be attributed to increased brightness due to methoxy-X04 accumulation.

YFP and methoxy-X04 were excited by a Ti:Sa laser (MaiTai, Spectra-Physics, Darmstadt, Germany) at 880 and $750 \mathrm{~nm}$ and the emission was collected from 527 to $582 \mathrm{~nm}$ and 460 to $500 \mathrm{~nm}$, respectively (LSM 5MP, Zeiss, Jena, Germany). A Zeiss $20 \times$ water-immersion objective (1.0 NA) was used to acquire image stacks of $425 \times 425 \times$ $300 \mu \mathrm{m}^{3}$ with $3 \mu \mathrm{m}$ z-resolution and $1,024 \times 1,024$ pixels per image frame $(0.41 \mu \mathrm{m} / \mathrm{pixel})$. In subsequent imaging sessions, previously imaged volumes were identified by 
eye, based on the positions of blood vessels, neuronal cell bodies and processes, as well as plaques. This allowed the precise alignment of the same imaging volume over a period of 6 weeks. The laser intensity was adjusted to keep the emitted YFP fluorescence stable.

Image processing and data analysis

The 3D images were deconvolved (AutoDeblur, Version x2.0.1, Media Cybernetics, Bethesda, USA) and contrastnormalized (i.e., based on the average and standard deviation of intensities of 3D stacks; Imaris 6.2.1, Bitplane, Zurich, Switzerland). For 3D analysis, we used surface rendering by Imaris software which also calculated the volume of individual plaques over time period. The 2D analysis was performed in maximum intensity projections applying a threshold of mean intensity + twice standard deviation of a representative background region. The volume growth of individual plaques was quantified as the slope of a linear fit of the volumes measured at each time point. We used repeated measures ANOVA and Student's $t$-test to test significance. All data are presented as mean $\pm \mathrm{SD}$, and $95 \%$ confidence intervals $(95 \% \mathrm{CI})$ are given where appropriate.

\section{Results}

We imaged 83 individual amyloid plaques in 12 months old Tg2576 mice [9] $(n=8)$ for up to 6 weeks (Fig. 1a). Volumes of individual plaques were three-dimensionally (3D) surface-rendered, quantified, and tracked over time (Fig. 1b). To compare the growth of plaques of various sizes, we normalized the volume of individual plaques to their volume at the first imaging session (week 0). In total, we detected the appearance of 25 new plaques during the imaging period of 6 weeks. Initially, these newborn plaques had an average volume of $255 \pm 231 \mu^{3}$ (95\% CI, 159-350 $\mu^{3}$; Fig. 2) and exhibited a significant volume increase of $2,015 \pm 1,783 \%$ over 6 weeks based on a linear regression (Fig. 3a-c; $P<0.001, R^{2}=0.97$ ). The initial area of newborn plaques, as determined from the maximum intensity projection, was $33.8 \pm 21.6 \mu \mathrm{m}^{2}(95 \%$ CI, 24.5-43.2 $\mathrm{mm}^{2}$ ) and they exhibited a significant area increase of $591 \pm 409 \%$ over 6 weeks based on a linear regression (Fig. 3d; $P<0.001, R^{2}=0.95$ ). Plaques that were not observed in their nascent stage, so called preexisting plaques $(n=58)$ had an initial average volume of $7,575 \pm 15,599 \mu^{3}$ (95\% CI, 3,473-11,676 $\mathrm{m}^{3}$; Fig. 2) and an initial average area, as determined from the

a

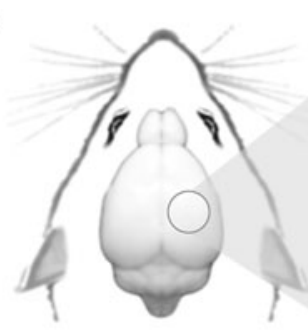

Tg2576 x YFP-H

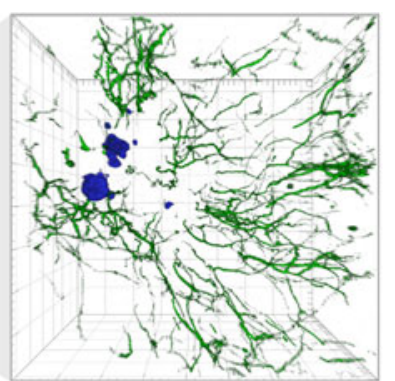

week 0

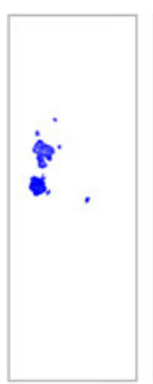

week 1

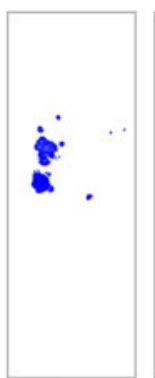

week 2

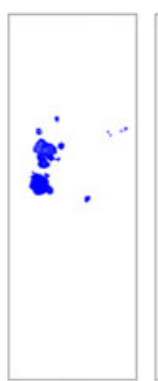

week 3

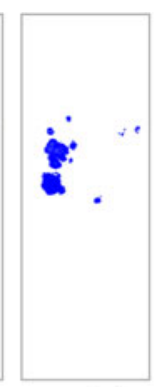

week 4

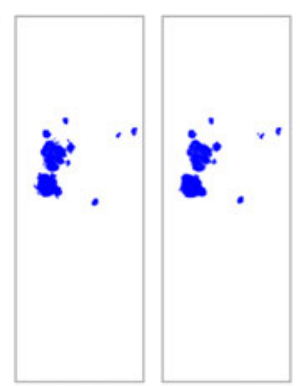

week 5

week 6
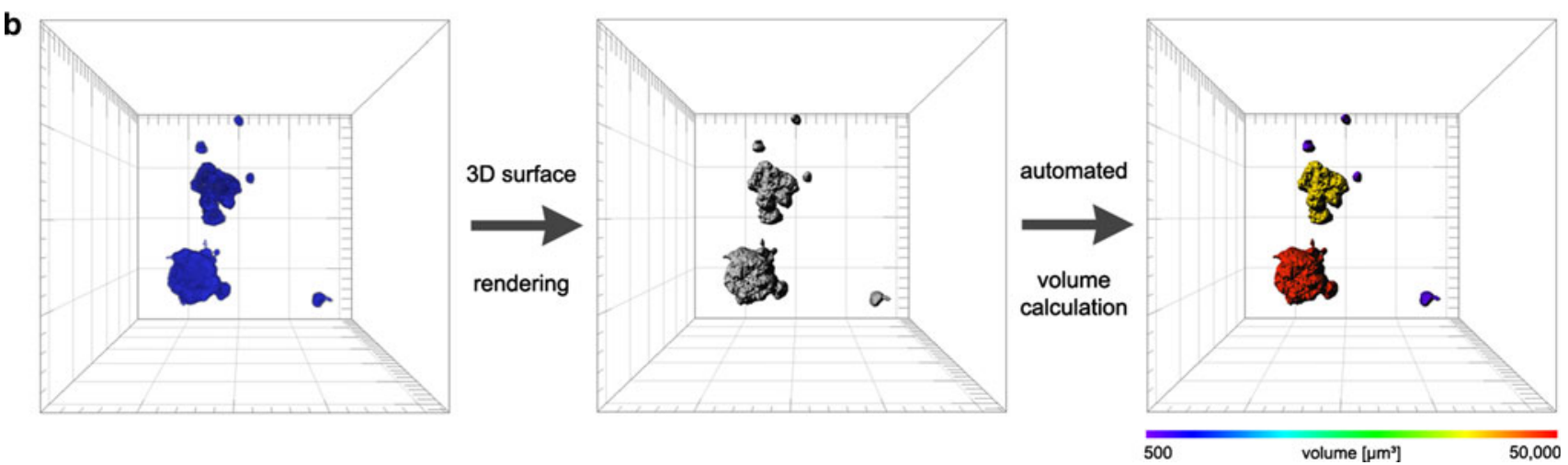

Fig. 1 Long-term two-photon in vivo imaging of amyloid plaques. a Schematic of a cranial window implanted over the somatosensory cortex of a Tg2576 mouse that was crossed with YFP-H. A volume rendered z-stack with perspective view $\left(45^{\circ}\right)$ shows amyloid plaques in blue (stained with methoxy-X04) and YFP-labeled dendrites in green at week 0 which was the first imaging time point. Amyloid plaques were followed over 6 weeks with weekly imaging time points illustrated as maximum intensity projections. b Illustration of the image analysis with Imaris software. After the amyloid plaques in the raw images were $3 \mathrm{D}$ surface rendered, the software automatically calculated the plaque volume which was then color-coded. Major ticks show $50 \mu \mathrm{m}$ 


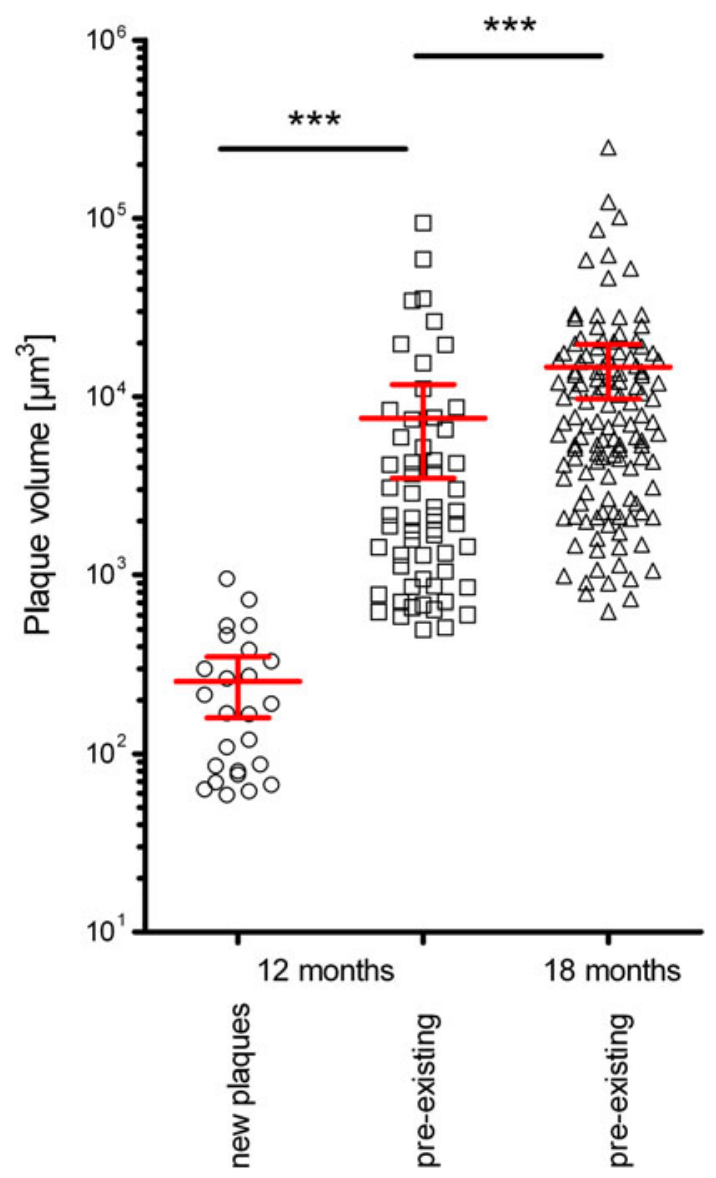

Fig. 2 Distribution of amyloid plaque volumes. Distribution of amyloid plaque volumes between individual newborn $(n=25)$ and pre-existing plaques $(n=58)$ in 12 months old $\operatorname{Tg} 2576$ and preexisting plaques $(n=128)$ at 18 months of age. The red bars show mean and 95\% CI. $* * * P<0.001$ (Kruskal-Wallis test with Dunn's post-hoc test)

maximum intensity projection, of $141.0 \pm 262.1 \mu \mathrm{m}^{2}$ (95\% CI, 73.3-208.7 $\mu \mathrm{m}^{2}$ ). Both volume as well as area was significantly higher compared to newborn plaques $(P<0.001)$. These pre-existing plaques exhibited a significant linear volume increase of $426 \pm 313 \%$ over 6 weeks (Fig. $4 \mathrm{a}-\mathrm{c}, P<0.001, R^{2}=0.99$ ), whereas the area increased by $282 \pm 123 \%$ over 6 weeks based on a linear regression (Fig. 4d; $P<0.001, R^{2}=0.97$ ). Collectively, all amyloid plaques analyzed in 12 months old $\operatorname{Tg} 2576$ mice significantly gained volume and area over 6 weeks $(P<0.001)$. In contrast, we neither detected newly appearing plaques, nor a significant increase in plaque volume in 18 months old Tg2576 mice over 6 weeks (Fig. $5 ; n=126$ plaques in three mice). At this age, amyloid plaques had an average volume of 14,632 \pm $27,811 \mu^{3}$ (95\% CI, 9,729-19,536 $\mu \mathrm{m}^{3}$; Fig. 2), which is significantly higher than at 12 months (Fig. $2, P<0.001$ ). We also determined the sphericity of the larger, preexisting plaques, which is defined as the ratio of the surface area of a sphere which has the same volume as the given plaque to the surface area of the plaque [22]. On average, the sphericity of $n=101$ plaques was $0.86 \pm 0.07$ (95\% CI, 0.84-0.87), which differed significantly from 1 (i.e., a perfect sphere; Wilcoxon signed rank test, $P<0.0001)$. Finally, we quantified the growth of individual plaques as the slope of a linear fit of the normalized volumes measured at each time point and plotted these against the initial volumes of each respective plaque (Fig. 6). This analysis shows a slight overlap in volumes of newborn and pre-existing plaques (Fig. 6a). This is most likely due to the sampling rate of plaque volumes (i.e., weekly), so that plaques labeled as "newborn" may have existed for up to a week before we detected them for the first time. More importantly, however, we found that in young (12 months) mice, plaque growth is directly related to the volume of a plaque (Fig. 6a), whereas most plaques in older (18 months) mice show very little, if any, growth (Fig. 6b).

\section{Discussion}

We monitored the appearance and growth of $\mathrm{A} \beta$ plaques in Tg2576 mice by in vivo two-photon microscopy of animals pretreated with methoxy-X04, which stains $\mathrm{A} \beta$ plaques. As a model, we used Tg2576 mice, which overexpress a human APP transgene containing the Swedish FAD mutation (K670N/M671L), and display the slowest amyloid pathology [9] of all the models analyzed by MeyerLuehmann et al. [16]. We reasoned that if we were able to detect amyloid plaque growth in this model, then it should also be detectable in other mouse models with a faster amyloid pathology.

In summary, we showed that in the early stages of amyloid pathology in $\operatorname{Tg} 2576$ mice, newborn plaques are initially small in size and grow over time. These findings are in contrast to the previous study by Meyer-Luehmann et al. [16] which found that plaques appeared very rapidly (within $24 \mathrm{~h}$ ) and did not change significantly in size thereafter. One possible reason for the differing results might be the fact that we primarily quantified volume, rather than area. However, even when we quantified plaque size as an area, that area steadily increased over 6 weeks (Figs. 3d, 4d). Thus, quantification of the plaques in three dimensions does not account for the observed difference. Another possibility is that open-skull surgery induces local inflammation and gliosis in the first weeks after surgery [8], which may affect plaque growth [24]. Therefore, in contrast to Meyer-Luehmann et al. [16], we waited for 3 weeks before imaging, so that possible inflammatory responses could abate. Yet even when we started daily imaging immediately after cranial window surgery, we were able to 
Fig. 3 Growth of newborn amyloid plaques in 12 months old Tg2576. a Maximum intensity projections of a time series of 3D images acquired with two-photon in vivo imaging in 12 months old Tg2576 mice. A newborn amyloid plaque (stained with methoxy-X04) is displayed in blue and YFP-labeled dendrites in green. This newborn plaque is not apparent at week 0 but emerges at week 2 . b In the following weeks, the nascent plaque considerably increased in size indicated by color-coded surface rendered objects. c The diagram shows normalized volumes of 25 individual newborn plaques (grey lines) based on the volume of first appearance. Week 0 is set as the time-point of first appearance. Average plaque volumes (red triangles), and linear regression of average plaque volume (red line) over 6 weeks are displayed. d The diagram shows normalized areas of 25 individual newborn plaques (grey lines) based on the area of first appearance. Week 0 is set as the time-point of first appearance. Average plaque areas (red triangles), and linear regression of average plaque volume (red line) over 6 weeks are displayed. Error bars show standard deviation. ***P $<0.001$; Scale bars show $10 \mu \mathrm{m}$
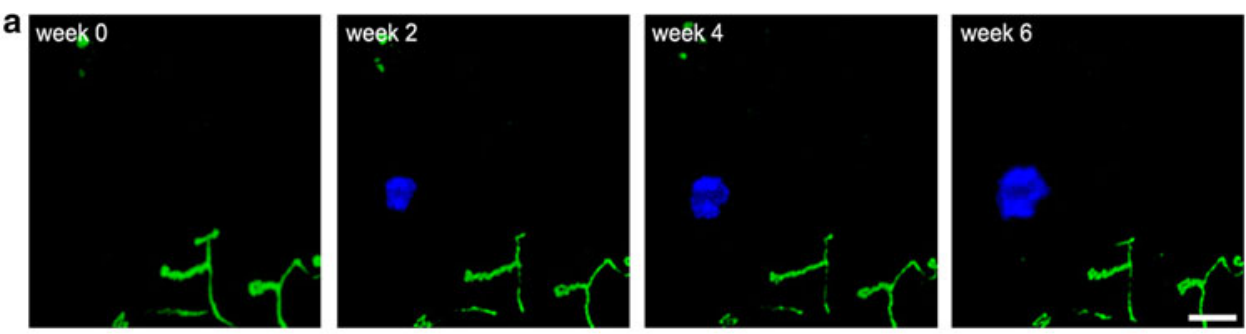

b



Volume $\left[\mu \mathrm{m}^{3}\right]$

20,000
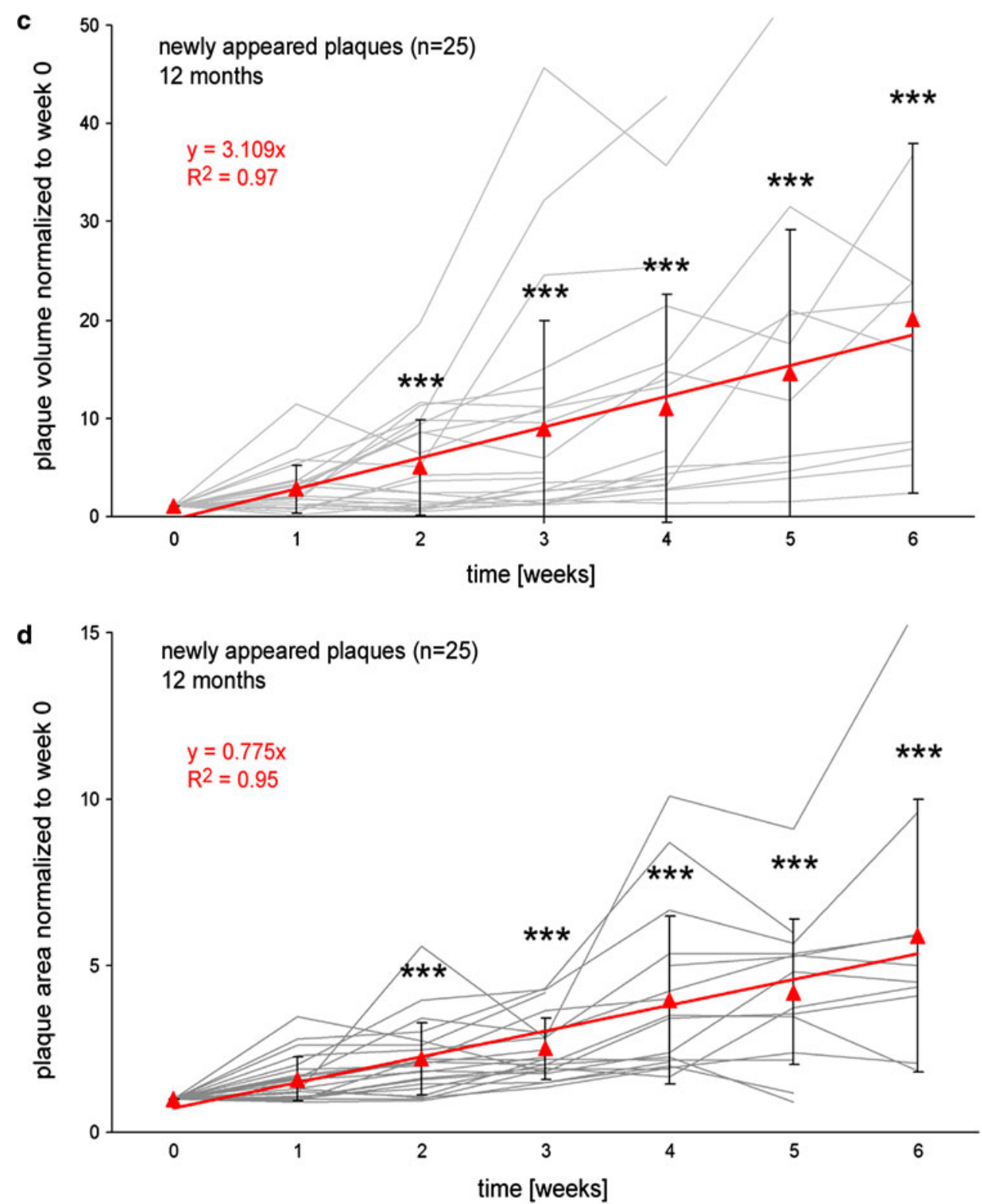

measure plaque growth. Initial plaque volumes were $92.1 \pm 64.5 \mu^{3} \quad$ (95\% CI, 60.0-124 $\left.\mu^{3} ; \quad n=18\right)$. However, a significant increase in plaque volume was only detected if we monitored plaque sizes for more than 16 days (Supplementary Fig. S2). Thus, delayed imaging 3 weeks after surgery does not account for the observed 
Fig. 4 Growth of pre-existing amyloid plaques in 12 months old Tg2576. a Maximum intensity projections of a time series of 3D images acquired with two-photon in vivo imaging in 12 months old $\operatorname{Tg} 2576$ mice. A pre-existing amyloid plaque (stained with methoxy-X04) is displayed in blue and YFP-labeled dendrites in green. This plaque is already apparent at week 0 . b In the following weeks, the plaque considerably increased in size indicated by color-coded surface rendered objects. c The diagram shows normalized volumes of 58 individual plaques (grey lines) based on the volume at the first imaging time-point (week 0). Average plaque volumes (red triangles), and linear regression of average plaque volume (red line) over 6 weeks are displayed. $\mathbf{d}$ The diagram shows normalized areas of 58 individual plaques (grey lines) based on the volume at the first imaging time-point (week 0).

Average plaque areas (red triangles), and linear regression of average plaque area (red line) over 6 weeks are displayed. Error bars show standard deviation. $* P<0.05$, $* * P<0.01, * * * P<0.001$; Scale bars show $10 \mu \mathrm{m}$
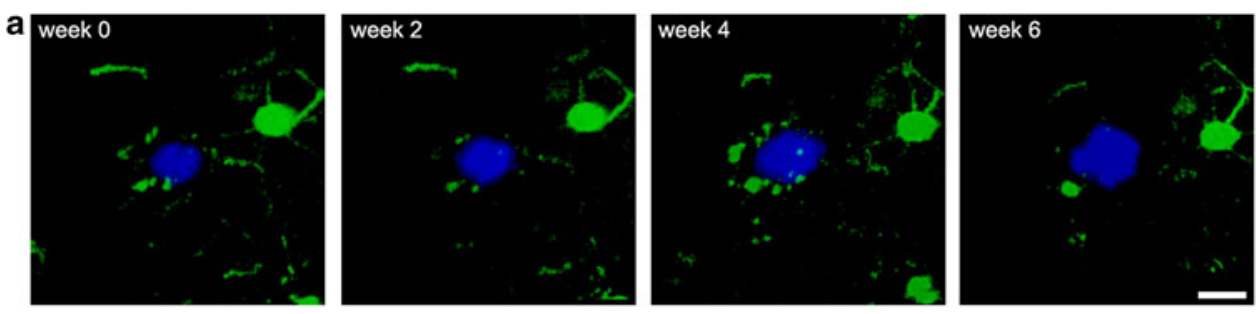

b
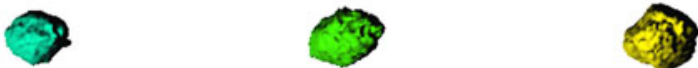

500

Volume $\left[\mu \mathrm{m}^{3}\right]$

20,000
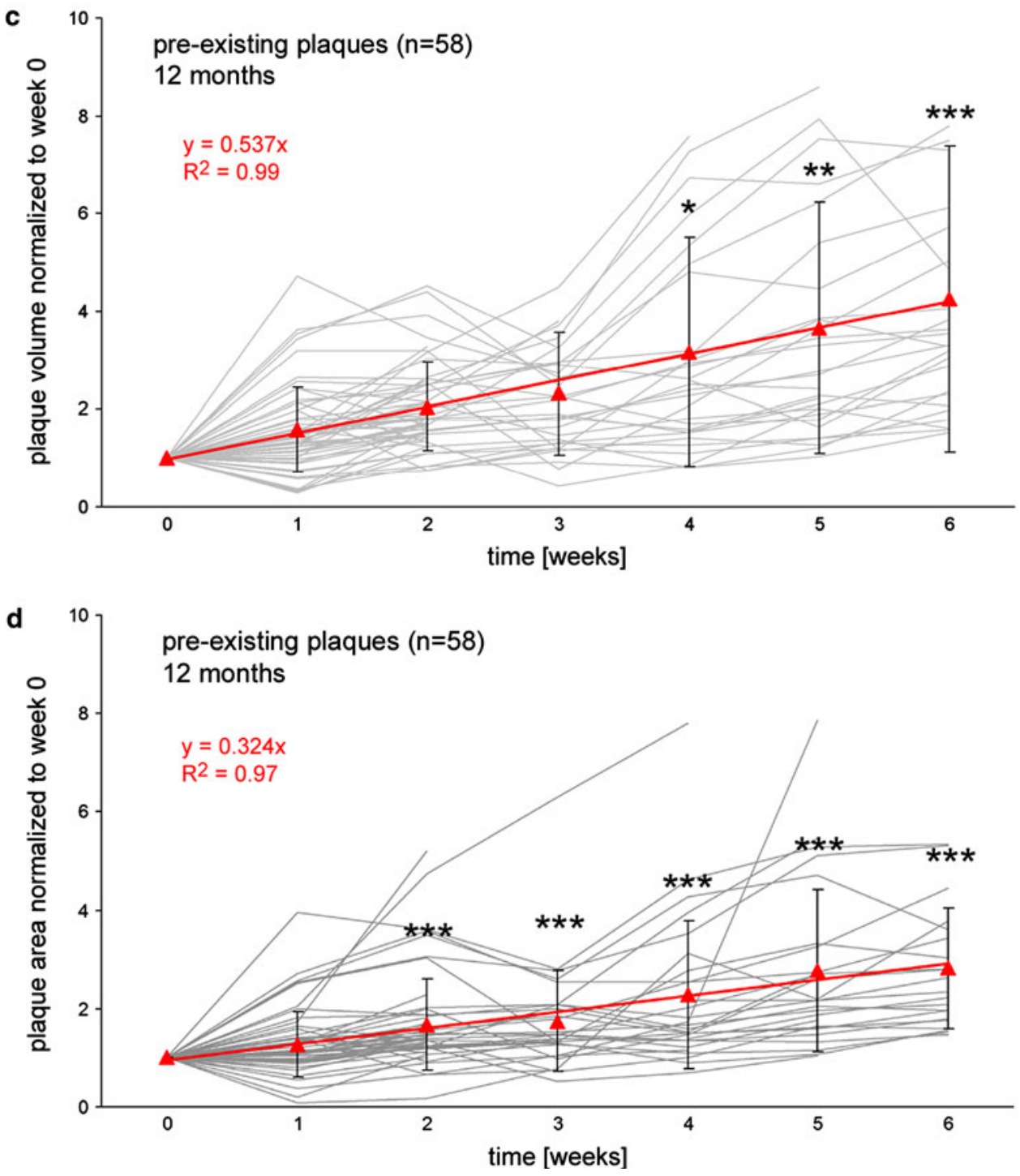

differences, either. We identified, however, two factors which determined if plaque growth can be observed in Tg2576 mice; first, and most importantly, we found that plaque growth can only be detected after a sufficiently long observation period. We monitored plaques for six consecutive weeks and detected that a statistically significant change in plaque volume of newborn plaques was achieved not earlier than 2 weeks after their first appearance (Fig. 3c), whereas a statistically significant change in plaque volume of pre-existing plaques was achieved not earlier than 4 weeks after the initial measurement (Fig. 4c). Second, we also found that the age of the observed animals 
Fig. 5 Growth of pre-existing amyloid plaques in 18 months old Tg2576. a Maximum intensity projections of a time series of 3D images acquired with two-photon in vivo imaging in 18 months old $\operatorname{Tg} 2576$ mice. A pre-existing amyloid plaque (stained with methoxy-X04) is displayed in blue and YFP-labeled dendrites in green. This plaque is already apparent at week 0 . b In the following weeks, the plaque does not increased in size indicated by color-coded diagram shows normalized volumes of 126 individual plaques (grey lines) based on the volume at the first imaging time-point (week 0). Average plaque volumes (red triangles), and linear regression of average plaque volume (red line) over 6 weeks are displayed. Error bars show standard deviation. Scale bars show $10 \mu \mathrm{m}$ surface rendered objects. c The


b
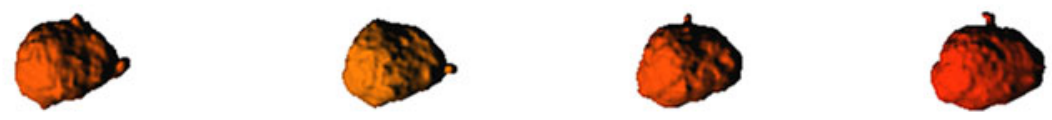

500

Volume $\left[\mu \mathrm{m}^{3}\right]$

20,000
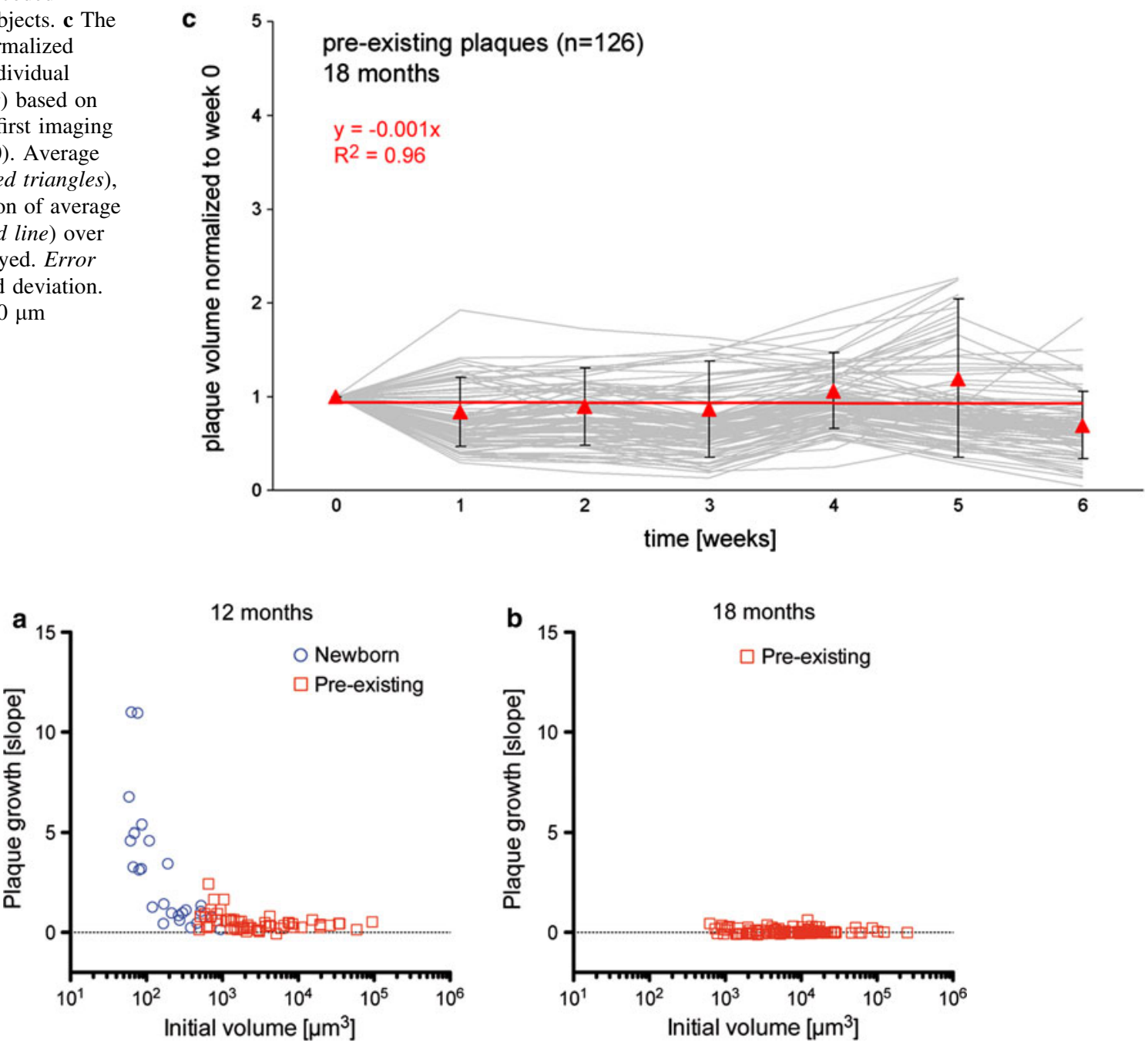

Fig. 6 Relationship between growth and initial volume of amyloid plaques. The growth of amyloid plaques is quantified as the slope of a linear fit of the normalized volumes measured at each time point and is shown as a function of the initial volume of plaques. a Data from

plays an important role; we detected plaque growth in 12 months old mice, whereas 18 months old mice showed no significant overall growth of plaques (Figs. 5c, 6),



12 months old animals. Newborn plaques are shown in blue and preexisting plaques in red. b Data from 18 months old animals. No newborn plaques were detected at that age. Pre-existing plaques are shown in red

which was shown before by Christie et al. [3]. Glial interaction with amyloid deposits has previously been suggested as a possible explanation for this observation in 
old mice [3]. However, we would further like to propose the possibility that the high number of existing amyloid plaques at this age and therefore the large total surface area where newly formed $\mathrm{A} \beta$ fibrils can attach to existing plaques could lead to a decelerated growth of each individual plaque up to a degree that it is not detectable by twophoton in vivo imaging anymore. It is furthermore interesting to note that plaque size varied widely in 18 months old animals (Fig. 2); yet we observed no newborn plaques at that age (Fig. 6). Individual plaques, however, seemed both to shrink and grow between measurements (Fig. 5). While this suggests the exciting possibility that plaques might be in a dynamic equilibrium, increasing measurement errors with increasing plaque size (note the heteroscedasticity in Figs. 3c, 4c) preclude us from making a firm conclusion. Thus, our data show that both the appropriate age and an appropriate observation period are required in order to detect plaque growth. Furthermore, our findings corroborate results which showed plaque growth in different double-transgenic $\mathrm{AD}$ mouse models $[2,24]$.

In conclusion, we show that amyloid plaques do grow in 12 months old Tg2576 mice when amyloid pathology is in the early stages [9]. Newborn amyloid plaques are initially small in size and grow over the following weeks. After the initial growth period, plaques continue to grow, albeit more slowly, until plaque growth is not detectable anymore in aged mice. These findings have important implications for AD diagnosis and therapy. A recent study [12], for instance, showed that in patients with mild cognitive impairment (MCI), amyloid plaque load, as measured by ${ }^{11} \mathrm{C}$-Pittsburgh compound $\mathrm{B}$ (PIB) retention in positron emission tomography, increased over 5 years, while the regional cerebral metabolic rate of glucose decreased. However, cognitive function remained stable in these patients. Patients with $\mathrm{AD}$, in contrast, had higher PIB retention than patients with MCI, which did not increase further over the observation period, and showed a decline in the regional cerebral metabolic rate of glucose as well as in cognitive function. In combination with our results, these data suggest that plaque formation occurs early in the course of the disease, but before a loss of cognitive function becomes apparent. Therefore, drugs that target plaque formation should be most effective early in the disease, when plaques are growing. These results also suggest that a different mechanism may be responsible for the cognitive decline in patients with manifest AD. Thus, an effort has to be made to find the mechanism of cognitive decline in the late disease stages in order to tailor effective therapeutic strategies.

Acknowledgments This work was supported by grants from the Deutsche Forschungsgemeinschaft (SFB 596, A13), the German Federal Ministry of Education and Research (Bundesministerium für Bildung und Forschung, 01GZ0713, 13N9268), the German Federal Ministry of Economics and Technology (Bundesministerium für
Wirtschaft und Technologie, 16IN0675) and the European Union (Neuro.GSK3, FP-7-223276). Furthermore, the authors would like to thank Sonja Steinbach for her excellent technical assistance and Patrizia Bonert for her invaluable assistance in animal care.

Open Access This article is distributed under the terms of the Creative Commons Attribution Noncommercial License which permits any noncommercial use, distribution, and reproduction in any medium, provided the original author(s) and source are credited.

\section{References}

1. Bittner T, Fuhrmann M, Burgold S et al (2009) gamma-Secretase inhibition reduces spine density in vivo via an amyloid precursor protein-dependent pathway. J Neurosci 29:10405-10409

2. Bolmont T, Haiss F, Eicke D et al (2008) Dynamics of the microglial/amyloid interaction indicate a role in plaque maintenance. J Neurosci 28:4283-4292

3. Christie RH, Bacskai BJ, Zipfel WR et al (2001) Growth arrest of individual senile plaques in a model of Alzheimer's disease observed by in vivo multiphoton microscopy. J Neurosci 21:858-864

4. Esler WP, Stimson ER, Ghilardi JR et al (1996) In vitro growth of Alzheimer's disease beta-amyloid plaques displays first-order kinetics. Biochemistry 35:749-757

5. Feng G, Mellor RH, Bernstein M et al (2000) Imaging neuronal subsets in transgenic mice expressing multiple spectral variants of GFP. Neuron 28:41-51

6. Fuhrmann M, Bittner T, Jung CKE et al (2010) Microglial CX3CR1 knockout prevents neuron loss in a mouse model of Alzheimer's disease. Nat Neurosci 13:411-413

7. Grutzendler J, Helmin K, Tsai J, Gan WB (2007) Various dendritic abnormalities are associated with fibrillar amyloid deposits in Alzheimer's disease. Ann NY Acad Sci 1097:30-39

8. Holtmaat A, Bonhoeffer T, Chow DK et al (2009) Long-term, high-resolution imaging in the mouse neocortex through a chronic cranial window. Nat Protocols 4:1128-1144

9. Hsiao K, Chapman P, Nilsen S et al (1996) Correlative memory deficits, Abeta elevation, and amyloid plaques in transgenic mice. Science 274:99-102

10. Hsieh H, Boehm J, Sato C et al (2006) AMPAR removal underlies Abeta-induced synaptic depression and dendritic spine loss. Neuron 52:831-843

11. Jarrett JT, Lansbury PT (1993) Seeding one-dimensional crystallization of amyloid-a pathogenic mechanism in Alzheimer's disease and scrapie. Cell 73:1055-1058

12. Kadir A, Almkvist O, Forsberg A et al (2010) Dynamic changes in PET amyloid and FDG imaging at different stages of Alzheimer's disease. Neurobiol Aging. doi:10.1016/j.neurobiolaging.2010.06. 015

13. Klunk WE, Bacskai BJ, Mathis CA et al (2002) Imaging Abeta plaques in living transgenic mice with multiphoton microscopy and methoxy-X04, a systemically administered Congo red derivative. J Neuropathol Exp Neurol 61:797-805

14. Knafo S, Alonso-Nanclares L, Gonzalez-Soriano J et al (2009) Widespread changes in dendritic spines in a model of Alzheimer's disease. Cereb Cortex 19:586-592

15. Koffie RM, Meyer-Luehmann M, Hashimoto T et al (2009) Oligomeric amyloid beta associates with postsynaptic densities and correlates with excitatory synapse loss near senile plaques. Proc Natl Acad Sci USA 106:4012-4017

16. Meyer-Luehmann M, Spires-Jones TL, Prada C et al (2008) Rapid appearance and local toxicity of amyloid-beta plaques in a mouse model of Alzheimer's disease. Nature 451:720-724 
17. Shankar GM, Li SM, Mehta TH et al (2008) Amyloid-beta protein dimers isolated directly from Alzheimer's brains impair synaptic plasticity and memory. Nat Med 14:837-842

18. Snyder EM, Nong Y, Almeida CG et al (2005) Regulation of NMDA receptor trafficking by amyloid-beta. Nat Neurosci 8:1051-1058

19. Spires TL, Meyer-Luehmann M, Stern EA et al (2005) Dendritic spine abnormalities in amyloid precursor protein transgenic mice demonstrated by gene transfer and intravital multiphoton microscopy. J Neurosci 25:7278-7287

20. Spires-Jones TL, Meyer-Luehmann M, Osetek JD et al (2007) Impaired spine stability underlies plaque-related spine loss in an Alzheimer's disease mouse model. Am J Pathol 171:1304-1311
21. Tsai J, Grutzendler J, Duff K, Gan WB (2004) Fibrillar amyloid deposition leads to local synaptic abnormalities and breakage of neuronal branches. Nat Neurosci 7:1181-1183

22. Wadell H (1935) Volume, shape, and roundness of quartz particles. J Geol 43:250-280

23. Wei W, Nguyen LN, Kessels HW et al (2010) Amyloid beta from axons and dendrites reduces local spine number and plasticity. Nat Neurosci 13:190-196

24. Yan P, Bero AW, Cirrito JR et al (2009) Characterizing the appearance and growth of amyloid plaques in APP/PS1 mice. J Neurosci 29:10706-10714 